\title{
COVID-19: a time of crisis, but also of surgical opportunity and optimism
}

The views expressed in this editorial are those of the author and do not necessarily reflect the position of the Canadian Medical Association or its subsidiaries.

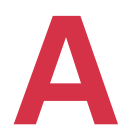
s of April 1, there are more than 850000 reported cases of coronavirus disease 2019 (COVID-19) during this global pandemic. It is also clear that both this denominator, as well as the casualty toll, will continue to rise. The widespread nature of this event is both unprecedented and unparalleled within our working lifetimes. Out of great pain, however, also comes great opportunity. More specifically, our exhausted Italian (and other) colleagues have reminded us of some of the very best qualities we can possess as surgeons: commitment, empathy, drive, collegiality, compassion and grace. The world needs more of these qualities in general, and hopefully this pandemic will provide a spark to revisit these behaviours in both our daily work and personal lives once things slow down again.

On a more concrete level, many of the experiences we take for granted as surgeons have come to a rapid halt. Predictable daily schedules, face-to-face social interactions with our colleagues, chatting with patients in outpatient clinics, and simply the efficient buzz of a busy hospital have all been greatly reduced. Although we contemplate many of these experiences with retrospective fondness in a world that has drastically changed during the past 3 weeks, new possibilities have also become clear. The forced transition to telehealth visits with patients has been remarkable. While the optimal platforms vary from region to region, and still require optimization with important issues such as security, confidentiality and efficiency, it is evident that patients (both remote and near) will demand this form of interaction in the future. As surgeons, we remain well positioned to lead the way in this form of patient-focused care. Similarly, the opportunity for video-based platforms in supporting our community and/or remote colleagues in trauma resuscitation, intubation, critical care and diagnosis in general is now at the forefront. Even technologies such as remote, nonexpert, hand-held tele-ultrasound may offer the interesting ability to diagnose and define early progression of pulmonary changes associated with COVID-19.1,2 Again, as surgeons, we have led the way with many of these remote technologies in both civilian and military applications and should continue to innovate, study and improve them on a rapid basis. This "once in a generation" opportunity to expand the reach of surgical systems to our most vulnerable patients must not be lost.

Research in particular displays a critical dichotomy in times of stress and uncertainty. While the severe acute respiratory syndrome (SARS) pandemic in 2003 and now
COVID-19 have confirmed the capacity of our peerreviewed journals and electronic communication to deliver rapidly evolving and relevant scientific information on diagnosis, treatment and outcomes, other areas of research have come to a stop. These areas include basic science bench-top work in many laboratories; approval of studies by research ethics boards from universities and health care regions; research support staff activities, such as patient recruitment for ongoing trials; and even submission processing and peer review at the journal level. Patience with all of our colleagues from both a multicentre investigator and local coordinator point of view will be essential. Having said that, a number of research groups throughout the country are already prospectively tracking the impact of COVID-19 on both elective surgical footprints, as well as emergency operative (and nonoperative) surgical volumes and outcomes. ${ }^{3}$

As surgeons, many of us are experienced in working within suboptimal conditions and challenging scenarios. Adaptability, perseverance, positivity and mission continue to describe the Canadian surgical community well in these unprecedented times. These qualities will be essential to ensuring that our patients, colleagues and families do as well as possible in this time of need. It is also our hope that you can find some support, or at the very least distraction, with the Cold Steel podcast (available at canjsurg.ca/podcasts and https://soundcloud.com/cjs-podcast), discussing topics from COVID-19 to leadership, artistic endeavours and surgical training.

Be well and continue to support each other at home, at work and in the community.

\section{Chad G. Ball, MD, MSc}

Coeditor, Canadian fournal of Surgery

Competing interests: None declared.

DOI: $10.1503 / c j s .003620$

\section{References}

1. Kirkpatrick AW. Point-of-care resuscitation research: from extreme to mainstream: Trauma Association of Canada Fraser Guard lecture 2019. 7 Trauma Acute Care Surg 2019;87:571-81.

2. Ball CG, Ranson MK, Rodriguez-Galvez M, et al. Sonographic depiction of posttraumatic alveolar-interstitial disease: the hand-held diagnosis of a pulmonary contusion. 7 Trauma 2009;66:962.

3. Ball CG, Murphy P, Verhoeff K, et al. A 30-day prospective audit of all inpatient complications following acute care surgery: How well do we really perform? Can 7 Surg 2020;63:E150-4. 